\title{
Datenschutz und Big Data?!?
}

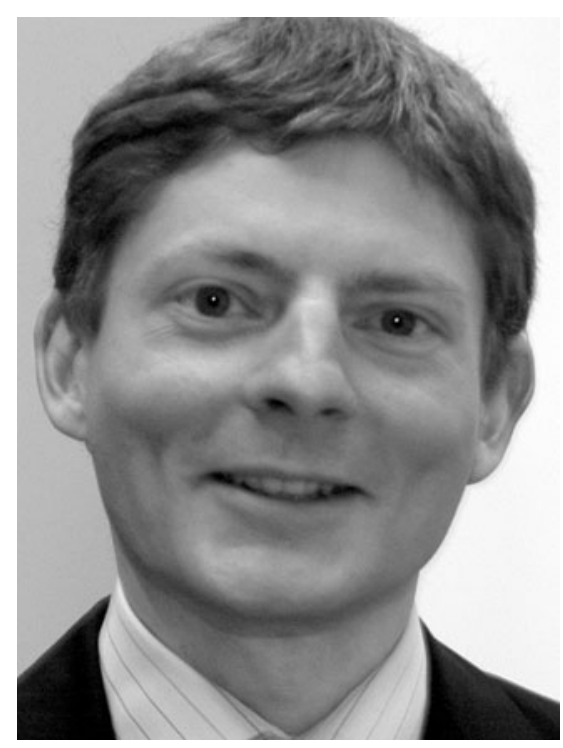

Bereits seit einigen Jahren macht nun das Schlagwort Big Data die Runde. Als Ausdruck für die Verarbeitung von bisher ungeahnten Datenmengen erschließt es sowohl im Bereich der Datenspeicherung und der Datenverarbeitung, aber auch der entsprechenden rechtlichen Grundlagen „Neuland“. Während es sich bei der Lösung der technischen Anforderungen eher um eine Frage der Zeit handeln wird, sieht es bei den rechtlichen Fragestellungen doch deutlich schwieriger aus.

Dies liegt unter anderem auch daran, dass bewährte Methoden, die Verarbeitung von „personenbezogenen“ Daten zu ermöglichen, bspw. indem diese vorher pseudonymisiert oder anonymisiert werden und damit nicht mehr unter den Anwendungsbereich des Datenschutzes fallen, im Zeitalter von Big Data nicht mehr funktionieren. Letztendlich kann man durchaus zu der Auffassung kommen, dass bei Big Data alle Daten als personenbeziehbar zu klassifizieren wären.

Den damit verbundenen Auswirkungen gehen die Autoren dieses Schwerpunktheftes anhand von konkreten Beispielen nach; sie zeigen dabei konkrete Problemfelder der Praxis auf und stellen zudem mögliche Lösungsansätze vor.

- Der erste Beitrag Das Ende der Anonymität von Volker Boehme-Neßler beleuchtet Big Data im Kontext der datenschutzrechtlichen Fragen - mit dem Ergebnis, dass das aktuelle Datenschutzrecht den Kontext Big Data offensichtlich nicht konsequent genug adressiert.

- Maik Sarunski beleuchtet in Big Data - Ende der Anonymität? die gleiche Fragestellung nunmehr aus Sicht einer Datenschutz-Aufsichtsbehörde. Anhand zahlreicher Beispiele illustriert er die Auswirkungen, die Big Data auf unseren Alltag haben kann und haben wird.

- Im Beitrag Anonymisierung, Pseudonymisierung und Transparenz für Big Data widmet sich Ninja Marnau der Frage, ob und wie die drei genannten Methoden im Rahmen von Big Data noch umsetzbar sind, und ordnet ihre Aussagen in den datenschutzrechtlichen Kontext der DSGVO ein.

- Verantwortlicher Einsatz von Big Data titelt der Beitrag von Oliver Raabe und Manuela Wagner. Sie beschreiben dabei Lösungsansätze, die Big Data nutzbar machen sollen, ohne dabei die Fragen des Daten- und vor allem auch des Persönlichkeitsschutzes zu vernachlässigen.

- Das Team rund um Martin Steinebach diskutiert im Beitrag Datenschutz und Datenanalyse die Herausforderungen bei der Datenanalyse unter Einsatz von Big Data-Methoden und zeigt dabei auch konkrete Lösungsansätze auf, mit denen Datenschutz und Datenanalyse vereint werden können.

- Ruth Stock-Homburg und Matthias Groß beleuchten das Thema Social Media Nutzung und Datensicherheit und gehen dabei der Frage nach, was bewährte Ansatzpunkte sind, um Social Media im Unternehmen als akzeptiertes Kommunikationsmedium zielführend einsetzen zu können.

Ergänzt wird der Schwerpunkt des Heftes diesmal durch zwei weitere Aufsätze:

- Volker Zeuner zeigt in seinem Beitrag Ein Modell für Datenschutz durch Datensicherheit auf, wie man aus seiner Sicht durch entsprechende organisatorische und technische Vorgaben die Kommunikation von Smart Metern absichern kann.

- Der Beitrag Der "Stand der Technik" im Kontext regulatorischer Anforderungen von Patrick Michaelis beschäftigt sich abschließend mit der Frage, was in der Praxis überhaupt der Anforderung Stand der Technik genügt.

Hinzu kommt diesmal noch ein Beitrag in der Rubrik „Forum“, der die Diskussion um eine Weiterentwicklung des Datenschutzrechts in Bezug auf Big Data anregen soll. Patrick Bunk und Patrick Goldschmidt illustrieren dazu im Beitrag Big Data und die Dual-Use Problematik am Beispiel öffentlicher Daten das komplizierte Verhältnis von innovativer Produktentwicklung und strikter Anwendung des Datenschutzrechts.

Zusammen mit dem gesamten Herausgeberteam wünsche ich Ihnen als Gastherausgeber eine informative und spannende Lektüre. Wir hoffen, dass auch diese Ausgabe Ihnen, verehrte Leserinnen und Leser, viele Anregungen für Ihre tägliche Praxis gibt.

\section{Christoph Wegener}

